\title{
Robust Tolerance Design of Bandpass Filter with Improved Frequency Response for Q-Band Satellite Applications
}

\author{
A. Sami, F. Teberio, L. Miranda, I. Arnedo, P. Martin-Iglesias, D. Benito, T. Lopetegi, M. A. G. Laso, \\ and I. Arregui
}

\begin{abstract}
A rectangular waveguide bandpass filter for Q-band with simple fabrication is proposed in this letter. The design is based on the use of the first passband replica of commensurateline stepped-impedance structures and achieves the suppression of their inherent low-pass response. In order to do it, the filter is implemented by rectangular waveguide sections with different widths and heights that can be analytically calculated. The technique is validated by a $9^{\text {th }}$ order Chebyshev filter with passband between 40 and $43 \mathrm{GHz}$ and fabrication yield equal to $84 \%$ for a manufacturing error of $\pm 20 \mu \mathrm{m}$. The measured results of the prototype fabricated with CNC milling are in good agreement with the simulated ones.
\end{abstract}

Index Terms-Rectangular waveguide, bandpass filter, fabrication tolerances, Q-band, satellite applications

\section{INTRODUCTION}

$\mathrm{N}$ EW trends in the space industry demand high data rates, better fabrication tolerances, and spurious free responses to have flawless communication between gateways and satellites. Space industry is exploring high frequency bands to increase communication capacity and, therefore, is interested in Q/V bands for commercial use [1], [2]. The typical solutions to design Q-band bandpass filters (BPFs) are based on the use of coupled resonant cavities. This concept is usually implemented by means of rectangular waveguide sections and inductive/capacitive irises [3]. When the classical Computer Numerical Control (CNC) milling is employed as the manufacturing method, the critical dimensions of these filters become comparable to the dimensions of the machining tools used [4], which may lead to consider highly precise CNC milling or the use of tuning screws to compensate manufacturing inaccuracies. This makes the manufacturing process more expensive and time consuming.

In order to improve fabrication tolerances, the first passband replica of the commensurate-line stepped-impedance low-pass filters can be employed to design BPFs [5]. Recently, this concept was used to design a BPF with relaxed tolerances for Q-band applications [6]. Low and high impedances were implemented by waveguide sections of equal width and different height to design the BPF. Although the fabrication

This paragraph of the first footnote will contain the date on which you submitted your paper for review. This work was supported by the Spanish Ministerio de Ciencia, Innovación y Universidades-Agencia Estatal de Investigación under Project TEC2017-85529-C3-2-R (AEI, FEDER-EU) and by the European Union's Horizon 2020 research and innovation programme under grant agreement no. 811232-TESLA-H2020-MSCA-ITN-2018. yield was remarkable, the inherent low-pass response of these structures can prevent their use in many applications.

In this letter, a method to design BPFs based on commensurate-line stepped-impedance structures is also proposed, but integrating section width changes to suppress the undesired low-pass response. Moreover, this advanced implementation will provide us with an extra degree of freedom to obtain initial and final dimensions equal to the standard port sizes, avoiding the use of the tapers typically required in these devices. Therefore, the performance and compactness of the filters designed in [6] will be highly improved.

\section{Design Method}

In the device proposed in [6], the first passband replica of a low-pass commensurate-line structure was employed to achieve a bandpass filter with reduced sensitivity to fabrication tolerances. However, the inherent low-pass response of this kind of structures will always be present and can even show up in the operating frequency range determined by the standard waveguide port employed in the filter, compromising its out-ofband behavior. This can be seen in Fig. 1, where the frequency behavior of a filter with constant width designed following [6] is depicted (dotted red line), and can be especially troublesome when designing filters with narrow bandwidth. In order to suppress the spurious low-pass response, a high-pass filter or another structure also designed using [6] but with a larger width and, therefore, the frequency behavior shown in Fig. 1 (dashed green line) may be concatenated to the previous one, resulting in the desired frequency response shown also in Fig. 1 (black line) but with a significant increment of the length. Therefore, the combination of commensurate-line sections with different widths in the same filter will be exploited in this method to suppress the spurious low-pass response. Moreover, the possibility of using different widths will permit to avoid the need of tapers, leading to more compact devices than in [6].

In order to use this concept to design bandpass filters with simple fabrication and suppression of the low-pass response, the next steps must be followed. Firstly, the order of the filter, $N$, the type of frequency response (all-pole), and the return loss in the passband (between the lower cut-off frequency, $f_{l}$, and

A. Sami, F. Teberio, I. Arnedo, D. Benito, T. Lopetegi, M. A. G. Laso, and I. Arregui are with the Electrical, Electronic, and Communications Engineering Department, Public University of Navarre, E-31006 Pamplona, Spain (e-mail: abdul.sami@unavarra.es).

P. Martin-Iglesias is with the European Space Agency ESA-ESTEC, 2201 AZ Noordwijk, The Netherlands. 
the upper cut-off frequency, $f_{2}$ ) must be specified. These frequencies correspond to electrical lengths of the commensurate-lines of $\pi-\theta_{c}$ and $\pi+\theta_{c}$, respectively, whereas $\pi$ is the electrical length at the central frequency of the passband, $f_{\pi}$, and $\theta_{c}$ can be calculated using (1) as it was explained in [7]:

$$
\theta_{c}=\pi \cdot \frac{\lambda_{f 1}-\lambda_{f 2}}{\lambda_{f 1}+\lambda_{f 2}}
$$

being $\lambda_{f i}$ the wavelength associated to the frequency $f_{i}$. Afterwards, the impedances $Z_{i}$ of the $N+2$ commensurate lines of the prototype that satisfies the specified all-pole filter response can be computed by applying the Richards' transformation and the extraction procedure explained in [8].

The implementation of these commensurate lines in rectangular waveguide technology is done by means of (2). As it is shown in [9], the characteristic impedance definition of (2) allows us to calculate the reflection coefficient at the junction between two waveguide sections assuming just $\mathrm{TE}_{10}$ singlemode operation. Only the effects of higher-order modes are neglected. Inspecting (2), the possibility to obtain a specific impedance using different values for the width $a_{i}$ and height $b_{i}$ of the waveguide section is revealed:

$$
Z_{i}=\frac{b_{i}}{\beta_{i}}=\frac{b_{i}}{\sqrt{\left(\frac{2 \cdot \pi \cdot f_{\pi}}{c}\right)^{2}-\left(\frac{\pi}{a_{i}}\right)^{2}}}
$$

where $c$ is the speed of light in vacuum and $f_{\pi}$ is the central frequency of the passband calculated as in [7]. In order to avoid glitches in the passband due to resonant higher-order modes in the larger cavities generated by manufacture imperfections and to obtain a gap easily fabricated with standard $\mathrm{CNC}$ milling in the smaller ones, the mapping of $Z_{i}$ with the physical dimensions of the waveguide sections must be done applying the following sequence: if $Z_{i}<Z_{i+1}$ then $b_{i}<b_{i+1}$ and $a_{i}>a_{i+1}$ whereas if $Z_{i}>Z_{i+1}$ then $b_{i}>b_{i+1}$ and $a_{i}<a_{i+1}$. Although other combinations are possible, this sequence leads to structures like the one shown in Fig. 2 where, according to (2), the height of the larger sections is reduced (to avoid glitches) due to the narrowing of their width and the height of the smaller ones can be increased (to simplify the fabrication) by broadening their width. Specifically, the largest impedance will be implemented by the section with the largest height $b_{\max }$ and the smallest width $a_{\min }$. Therefore, a value smaller than the waveguide standard port must be fixed for $a_{\min }$, and then $b_{\max }$ can be obtained using (2). It is important to note that these values should fulfill (3) to avoid possible glitches in the passband due to the higher-order $\mathrm{TE}_{110}$-mode resonance [8]:

$$
b_{\text {max }} \leq \frac{1}{\sqrt{\left(\frac{2 \cdot f_{2}}{c}\right)^{2}-\frac{1}{\left(a_{\min }\right)^{2}}}}
$$

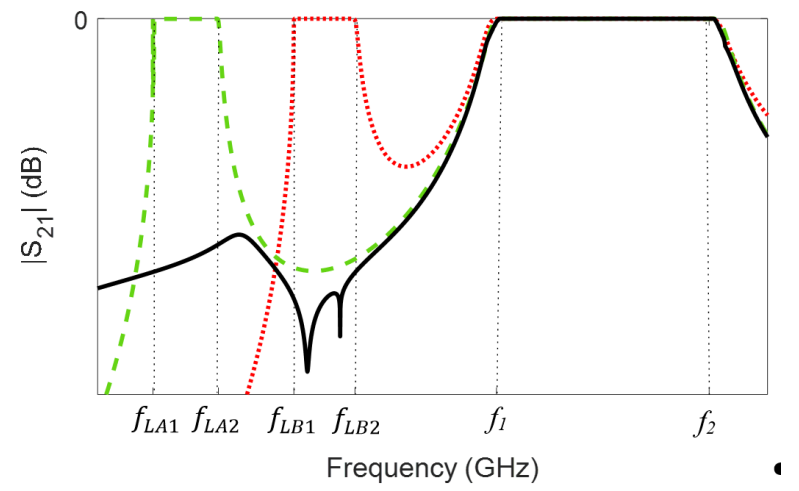

Fig. 1. Frequency response of a filter designed with constant width equal to $a_{\min }$ (dotted red line), a filter designed with constant width equal to $a_{\max }$ (dashed green line), and the structure formed by concatenating both filters (black line).

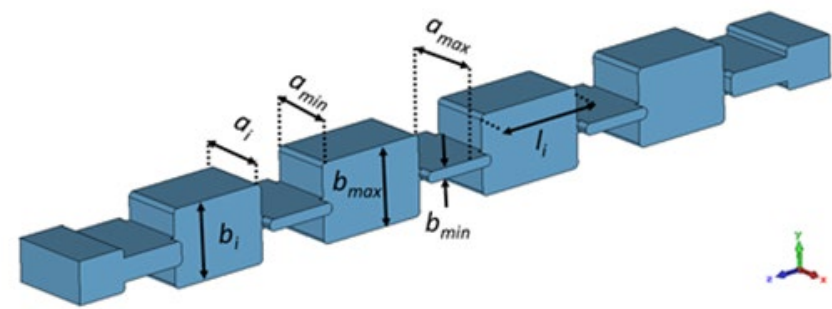

Fig. 2. 3D schematic of a filter designed with the proposed method (including rounded corners for fabrication by $\mathrm{CNC}$ milling).

Once $a_{\min }$ and $b_{\max }$ are fixed, the width of the smallest section $a_{\max }$ (which will implement the lowest impedance value) can be determined to fulfill $f_{L B 1}>f_{L A 2}$ (see Fig. 1), where $f_{L A 2}$ is the highest frequency of the spurious low-pass response associated with the filter with constant width equal to $a_{\max }$ designed following [6] and $f_{L B 1}$ is the lowest frequency of the spurious low-pass response associated with the filter with constant width equal to $a_{\min }$ designed following [6]. The height of the smallest section, $b_{\min }$, can be calculated afterwards using (2). Finally, considering $a_{\min }$ and $a_{\max }$ for the larger and smaller sections, respectively, the remaining heights of the sections $b_{i}$ can be also obtained using (2).

After the calculation of the heights $b_{i}$ and widths $a_{i}$ of each section, their initial lengths $l_{i}=\lambda_{f \pi} / 2$ must be modified to compensate for the phase variation produced by the effect of the higher-order modes at the consecutive section junctions. In order to do it, the phase conditions for the $S_{11}$ and $S_{21}$ parameters must be satisfied and the length $l_{i}$ of each section will be modified to restore them as detailed in [7]. A final optimization might be done, if needed, to adjust the return loss parameter.

\section{DESIGN EXAMPLE}

The technique explained in Section II will be used to design a BPF for Q-band. The design example will have a passband between $f_{1}=40 \mathrm{GHz}$ and $f_{2}=43 \mathrm{GHz}$ with in-band return loss better than $20 \mathrm{~dB}$. The required out of band attenuation will be $50 \mathrm{~dB}$ between $33-38 \mathrm{GHz}$ and $46.5-50 \mathrm{GHz}$. The size of the input/output ports will be equal to the standard WR22 $\left(a_{l}=5.69 \mathrm{~mm}, b_{I}=2.845 \mathrm{~mm}\right)$.

\section{A. Design and Simulation}

In order to include frequency margins, a $9^{\text {th }}$ order Chebyshev filter with a passband between 39.5 to $43.5 \mathrm{GHz}$ and return loss 
equal to $25 \mathrm{~dB}$ will be designed. Following [6] and considering a constant width equal to $4.25 \mathrm{~mm}$, the frequency response shown in Fig. 3 (green line) will be obtained (the normalized impedance values for the required specifications are: $Z_{I}=Z_{11}=1$, $Z_{2}=Z_{10}=0.58, \quad Z_{3}=Z_{9}=2.32, \quad Z_{4}=Z_{8}=0.32, \quad Z_{5}=Z_{7}=2.92, \quad$ and $Z_{6}=0.30$ and the resulting dimensions are given in Table I). Although the in-band specifications are fulfilled, the inherent low-pass response is close to the passband, being not possible to achieve the required attenuation in the stopband. Moreover, this filter requires a taper in each side of the filter to match the initial height $b_{0}=b_{11}=1 \mathrm{~mm}$ and width $(4.25 \mathrm{~mm})$ with the standard WR22 waveguide ports. With the technique proposed in this paper, the low-pass response will be suppressed and standard ports will be obtained without using tapers, achieving a length reduction equal to $30 \%$.

Following the method explained in the previous section, the minimum width, which corresponds to the largest normalized impedance $\left(Z_{5}=Z_{7}=2.92\right)$ is fixed to $a_{\min }=4.25 \mathrm{~mm}$, a value smaller than the waveguide standard port width. Then using (2), $b_{\max }=5.62 \mathrm{~mm}$ is obtained, which avoids the possibility of having glitches in the passband due to the higher-order $\mathrm{TE}_{110^{-}}$ mode resonance since (3) is satisfied, $b_{\max } \leq 5.88 \mathrm{~mm}$. As a filter with constant width equal to $a_{\min }=4.25 \mathrm{~mm}$ presents a low-pass response from $f_{L B 1}=35.29 \mathrm{GHz}$ to $f_{L B 2}=35.53 \mathrm{GHz}$ (see Fig. 3), the width of the smallest section is set to $a_{\text {max }}=5.9 \mathrm{~mm}$ since its associate low-pass response is up to $f_{L A 2}=25.57 \mathrm{GHz}$, clearly fulfilling that $f_{L B 1}>f_{L A 2}$. Afterwards, the height values of the other sections are obtained using (2), employing $a_{\min }$ for the larger waveguide sections and $a_{\max }$ for the smaller ones. Finally, the lengths are adjusted to satisfy the $S_{I I}$ and $S_{2 l}$ phase conditions given in [7]. In order to fabricate the filter by $\mathrm{CNC}$ milling, rounded corners with a radio equal to $0.3 \mathrm{~mm}$ have been included in the structure and the dimensions have been slightly optimized to compensate the effects (final dimensions considering rounded corners shown in Table II). The CST MWS simulated frequency response is shown in Fig. 3 (red line).

\section{B. Sensitivity Analysis}

The sensitivity analysis of the filter is performed using FEST3D. All dimensions of the filter are varied using a standard deviation value of $8 \mu \mathrm{m}$, which will vary the dimensions of the filter up to $20 \mu \mathrm{m}$. One hundred iterations have been performed, obtaining an outstanding manufacturing yield of $84 \%$ in the novel filter whereas the manufacturing yield of the classic inductive iris filter designed to fulfill the same specifications was only $12 \%$ (see Fig. 4).

\section{Fabrication and Measurement}

The novel filter is fabricated using CNC milling in bare aluminum (see Fig. 3). The measured frequency response shown in Fig. 3 (black line) shows good agreement with the simulated one and confirms that the spurious low-pass response is fully suppressed. No tuning elements (usually needed in Qband filters) have been necessary to achieve the required return loss and the insertion loss parameter may be improved, if needed, by silver plating the prototype. Specifically, a bandpass
Table I. Dimensions of the BPF designed following [6] with $a_{0}=4.25(\mathrm{~mm})$

\begin{tabular}{cccccc}
\hline \hline$b_{1}=b_{11}$ & $b_{2}=b_{10}$ & $b_{3}=b_{9}$ & $b_{4}=b_{8}$ & $b_{5}=b_{7}$ & $b_{6}$ \\
1 & 0.60 & 2.26 & 0.33 & 2.91 & 0.31 \\
\hline \hline$l_{1}=l_{11}$ & $l_{2}=l_{10}$ & $l_{3}=l_{9}$ & $l_{4}=l_{8}$ & $l_{5}=l_{7}$ & $l_{6}$ \\
3.8 & 6.64 & 6.99 & 6.54 & 6.97 & 6.53 \\
\hline \hline
\end{tabular}

Table II. Dimensions of the final design with rounded corners (mm)

\begin{tabular}{cccccc}
\hline \hline$a_{1}=a_{11}$ & $a_{2}=a_{10}$ & $a_{3}=a_{9}$ & $a_{4}=a_{8}$ & $a_{5}=a_{7}$ & $a_{6}$ \\
5.69 & 5.69 & 4.27 & 5.83 & 4.13 & 5.86 \\
\hline \hline$b_{1}=b_{11}$ & $b_{2}=b_{10}$ & $b_{3}=b_{9}$ & $b_{4}=b_{8}$ & $b_{5}=b_{7}$ & $b_{6}$ \\
2.84 & 1.57 & 5.17 & 0.764 & 5.31 & 0.73 \\
\hline \hline$l_{1}=l_{11}$ & $l_{2}=l_{10}$ & $l_{3}=l_{9}$ & $l_{4}=l_{8}$ & $l_{5}=l_{7}$ & $l_{6}$ \\
3.5 & 4.47 & 6.84 & 4.88 & 7.59 & 4.97 \\
\hline \hline
\end{tabular}

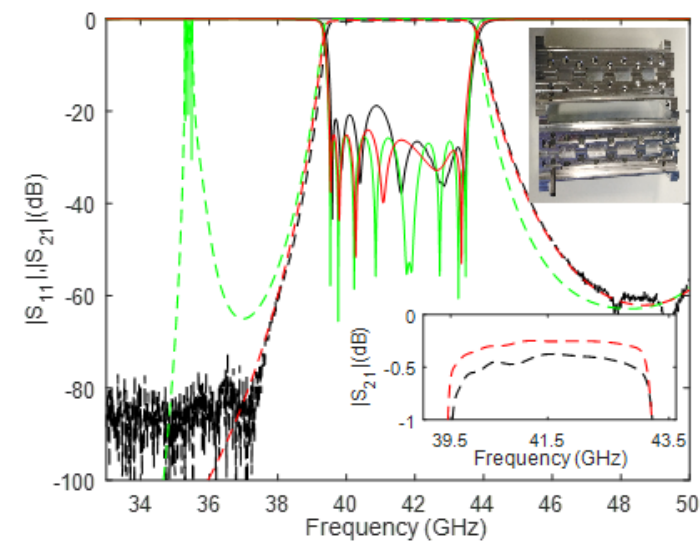

Fig. 3. CST MWS simulated frequency response of the filter with constant width designed following [6] (green line) and the novel design with suppression of the low-pass response including rounded corners (red line). Measured response of the prototype (black line). $\left|S_{11}\right|$ in solid line and $\left|S_{21}\right|$ in dashed line. Inset: detail of the insertion loss and photograph of the unassembled prototype.

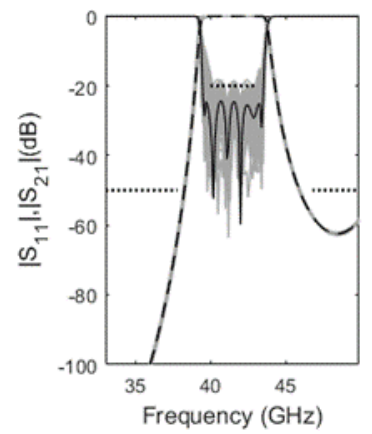

(a)

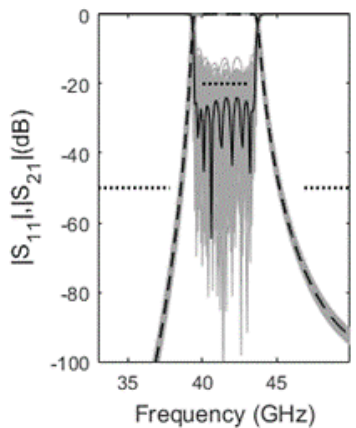

(b)
Fig. 4. Sensitivity analysis of (a) the proposed design, (b) the classic inductive iris filter. $\left|S_{11}\right|$ in solid line, $\left|S_{21}\right|$ in dashed line. Black line for the baseline filter and grey line for the simulation trials. Frequency specifications in dotted line.

response centered at $41.5 \mathrm{GHz}$, with a bandwidth equal to $3 \mathrm{GHz}$, in-band return loss better than $20 \mathrm{~dB}$, and insertion loss equal to $0.37 \mathrm{~dB}$ at the center frequency is obtained.

\section{CONCLUSION}

A novel filter design methodology has been proposed by cascading waveguide sections with varying widths and heights to achieve a bandpass response with straightforward fabrication and the suppression of the undesired low-pass response of this kind of structures. The technique has been validated with a $\mathrm{Q}-$ band device with passband between $40 \mathrm{GHz}$ and $43 \mathrm{GHz}$, showing an excellent fabrication yield. A prototype has been fabricated and measured validating the expected frequency response. 


\section{REFERENCES}

[1] J. V. Evans and A. Dissanayake, "Prospects for commercial satellite services at Q- and V-bands," in Proc. IEEE Military Communications Conference (MILCOM 98), Boston, MA, USA, 1998, pp. 1-7.

[2] A. Martellucci, J. R. Castro, P. Sivac, and E. Benzi, "The Alphasat Aldo Paraboni Scientific experiment: an overview of the activities of the European Space Agency," in Proc. $10^{\text {th }}$ Eur. Conf. on Antennas and Prop., Davos, 2016, pp. 1-4.

[3] V. E. Boria and B. Gimeno, "Waveguide filters for satellites," in IEEE Microwave Magazine, vol. 8, no. 5, pp. 60-70, Oct. 2007.

[4] P. Soto, V. E. Boria, C. Carceller, C. P. Vicente, J. Gil, and B. Gimeno, "EM-based synthesis and design of bandpass waveguide filters including manufacturing effects with FEST 3D," Int. J. RF and Microwave CAE, vol. 22, no. 1, pp. 93-103, Jun. 2012.

[5] G. L. Matthaei, L. Young, and E. M. T. Jones, "Microwave filters, impedance-matching networks, and coupling structures," vol. 5, New York: McGraw-Hill, 1964.

[6] F. Teberio, P. Soto, I. Arregui, T. Lopetegi, S. Cogollos, I. Arnedo, P. Martin-Iglesias, V. E. Boria, and M. A. G. Laso, "Waveguide band-pass filter with reduced sensitivity to fabrication tolerances for Q-Band Payloads," in IEEE MTT-S International Microwave Symposium Conference (IMS), USA, 2017, pp. 1464-1467.

[7] F. Teberio, J. M. Percaz, P. Iglesias, I. Arregui, P. Martin-Iglesias, I. Arnedo, T. Lopetegi, and M. A. G. Laso, "Rectangular waveguide filters with meandered topology," IEEE Transactions on Microwave Theory and Techniques, vol. 66, no. 8, pp. 3632-3643, 2018.

[8] R. J. Cameron, C. M. Kudsia, and R. R. Mansour, "Microwave Filters for Communication Systems: Fundamentals, Design and Applications.," Hoboken, NJ: John Wiley \& Sons, 2007.

[9] B. Z. Katsenelenbaum, L.Mercader, M. Pereyaslavets, M. Sorolla, and M. Thumm, Theory of Nonuniform Waveguides-The Cross Section Method, series IEE Electromagnetic Waves 44. London, U.K.: IEE Press, 1998. 